CARVALHO, J.O.M.; LUZ, J.M.Q.; JULIATTI, F.C.; MELO, L.C.; TEODORO, R.E.F.; LIMA, L.M.L. Desempenho de famílias e híbridos comerciais de tomateiro para processamento industrial com irrigação por gotejamento. Horticultura Brasileira, Brasília, v. 21, n. 3, p. 525-533, julho-setembro 2003.

\title{
Desempenho de famílias e híbridos comerciais de tomateiro para processamento industrial com irrigação por gotejamento ${ }^{1}$
}

\author{
José O.M. Carvalho²; José M.Q. Luz ${ }^{3}$; Fernando C. Juliatti³; Leonardo C. Melo ${ }^{4}$; Reges E.F. Teodoro ${ }^{3}$; \\ Lais M.L. Lima ${ }^{5}$ \\ ${ }^{2}$ Embrapa Rondônia, C. Postal 406, 78900-970 Porto Velho-RO; E-mail: orestes@cpafro.embrapa.br; ${ }^{3}$ UFU, ICIAG, C. Postal 593, \\ 38400-902 Uberlândia-MG; ${ }^{4}$ Embrapa Arroz e Feijão, C. Postal 179, 75375-000 Santo Antônio de Goiás-GO; ${ }^{5}$ Faculdade São Lucas, \\ 78916-450 Porto Velho-RO
}

\section{RESUMO}

Foram avaliadas e selecionadas as famílias provenientes do programa de melhoramento do tomateiro da UFU, com potencial para processamento industrial e comparados seus desempenhos com os híbridos mais utilizados pelos produtores na região de Patos de Minas. O ensaio foi conduzido na EE Fazenda Barreiro da Unilever Bestfoods Brasil, em Patos de Minas. Utilizaram-se 30 famílias de tomateiro e os híbridos Heinz 9553, Heinz 7155N2 e Hypeel 108. As Parcelas constituíram-se de linhas duplas espaçadas $0,5 \mathrm{~m}$ entre si, com $10 \mathrm{~m}$ de comprimento, irrigadas por gotejamento. O espaçamento entre as linhas duplas foi de $1,3 \mathrm{~m}$ e a população de 27 mil plantas por hectare. $\mathrm{O}$ delineamento experimental foi de blocos ao acaso, com quatro repetições. A semeadura foi realizada em 19/05/01. O rendimento de polpa mostrou correlações fenotípicas e genotípicas positivas com a produção total, produção de frutos comerciais, \% de sólidos solúveis totais, coloração do suco, número de frutos maduros por planta e $\mathrm{pH}$ e negativa com acidez total titulável. Destacaram-se a família UFU 06 e os três híbridos comerciais.

Palavras-chave: Lycopersicon esculentum, cultivares, processamento, melhoramento, correlação.

\begin{abstract}
Evaluation of tomato families and commercial hybrids for processing using drip irrigation system

Tomato families originated from the processing tomato breeding program of the Universidade Federal de Uberlandia were evaluated and selected and compared to the performance with the most planted hybrids in Patos de Minas, Brazil. The experiment was conducted in the E. E. Barreiro, Unilever Bestfoods, in Patos de Minas. Thirty tomato families and the hybrids Heinz 9553, Heinz 7155N2 and Hypeel 108 were used. Experimental plots consisted of two lines, each one $10 \mathrm{~m}$ long, spaced $0,5 \mathrm{~m}$ from each other and irrigated with drip irrigation. The space between each double row was $1,3 \mathrm{~m}$ and the population of 27 thousand plants per hectare. The experimental design was a randomised complete block with four repetitions, installed on $05 / 19 / 01$. The paste yield showed positive phenotypic and genotypic correlation with total fruit yield, commercial fruit yield, percentage of total soluble solids, juice coloration, number of mature fruits per plant and $\mathrm{pH}$ and negative correlation with the total titled acidity. Best results were observed in the family UFU 06 and in the commercial hybrids.
\end{abstract}

Keywords: Lycopersicon esculentum, cultivars, processing, breeding, correlation.

\section{(Recebido Para publicação em 18 de junho de 2002 e aceito em 22 de abril de 2003)}

$\mathrm{O}$ tomate não é uma das hortaliças mais ricas em vitaminas e sais minerais, especialmente por conter, em média, $94 \%$ de água no fruto ao natural. No entanto, por ser consumido em maior quantidade, com maior frequência em relação a outras hortaliças e seu consumo ser feito em grande parte sem a cocção, o tomate torna-se uma importante fonte de vitaminas e sais minerais na dieta do brasileiro, como, por exemplo, de vitamina $C$, cujo teor varia de 11,2 a $21,6 \mathrm{mg} / 100 \mathrm{~g}$ de frutos e das vitaminas $\mathrm{A}, \mathrm{B}_{1}, \mathrm{~B}_{2}, \mathrm{P}$ e K. O tomate contém outras substâncias, em doses mínimas, porém muito importantes, a começar pelas substâncias corantes licopeno (vermelho) e caroteno (amarelo). Folhas e frutos ainda verdes também possuem uma substância levemente tóxica, o alcalóide tomatina, que parece eficaz contra fungos de micoses da pele humana (Filgueira, 2000).

A cultura do tomateiro possui grande importância econômica pelo volume e valor da produção. O mercado brasileiro de tomate industrial de cultivo rasteiro, destinado à produção de purês, molhos prontos, extratos, sucos, etc., é avaliado em US\$ 445 milhões anuais (Nakamae \& Pastrello, 1999). O consumo nacional de polpa tem aumentado com o crescimento das redes de "fastfood" e o ingresso de novas indústrias de molhos prontos. O consumo de polpa de tomate passou de $95 \mathrm{mil} \mathrm{t}$, em
1994, para 140 mil t em 1998 (Nakamae \& Pastrello, 2000). No Brasil, a produção de tomate para processamento industrial, no último quinquênio, girou em torno de 1 milhão de t/ano, com a marca recorde de 1,29 milhão de toneladas alcançada na safra de 1999 (Melo, 2001). A região do Cerrado transformou-se na mais importante zona de produção de tomate para processamento industrial do país, com $77 \%$ da área plantada, seguida do estado de São Paulo com $14 \%$ e da região Nordeste, com apenas 9\% (Melo, 2001).

Nos últimos dez anos, a maioria das plantações de tomate para processamento industrial tem sido irrigada por aspersão, especialmente por

\footnotetext{
${ }^{1}$ Extraído da dissertação de mestrado do primeiro autor (Carvalho, 2002), realizada com apoio da CAPES.
} 
pivô central, tendo alcançado produtividades acima de 75 t/ha. Em decorrência da falta de um bom manejo de água, falta de rotação de culturas e ocorrência severa de doenças, os produtores vêm procurando sistemas de irrigação alternativos. Trabalhos experimentais têm mostrado que a irrigação por gotejamento, também poderá ser uma opção (Silva \& Marouelli, 1995). O grande interesse atual por gotejamento na tomaticultura industrial foi despertado principalmente pelos excelentes resultados de economia de água e energia, aliados a um substancial aumento de produtividade e melhoria da qualidade da matéria-prima. Phene (1999) relatou que, em pesquisas com tomate para processamento (Var. UC-82B) na Califórnia, foram alcançadas produtividades total acima de $200 \mathrm{t} /$ ha e comercial acima de $145 \mathrm{t} / \mathrm{ha}$.

Segundo Zahara (1970), Rick (1978), maluf(1994) e Melo (2001), em trabalhos de melhoramento de tomateiros visando processamento industrial deve-se buscar a obtenção de plantas compactas, pequenas, com maturação concentrada dos frutos visando colheita mecanizada, produtivas e resistentes às doenças. A agroindústria exige um tipo especial de tomate, obrigatoriamente produzido em cultura rasteira, sem tratos culturais sofisticados, objetivando baixo custo de obtenção da matéria-prima (Filgueira, 2000). Desta forma, durante o processo de seleção de genótipos para colheita mecanizada devem ser avaliados prioritariamente: a concentração de maturação, o potencial produtivo, o tamanho da rama que deve ser mediano, a cobertura dos frutos, a capacidade de permanência dos frutos na planta, a firmeza que permita o transporte dos frutos a granel e o índice de retenção de pedúnculo. Outras características inerentes às cultivares que se destinam ao processamento industrial como o teor de sólidos solúveis $\left({ }^{\circ} \mathrm{Brix}\right.$ acima de 5,0), coloração vermelha intensa (externa e interna), pericarpo espesso, inserção peduncular pequena, ausência de defeitos (ombro verde, coração negro, "zippering", "split setting") e resistência às doenças que causam prejuízo à cultura, entre outras, devem ser cuidadosamente avaliadas durante o processo de seleção (Melo, 2001).
Neste sentido, a UFU, com apoio do CNPq e da CAPES, vem desenvolvendo, desde 1995, o Programa de Melhoramento Genético do Tomateiro, a partir de cruzamentos entre os parentais BHRS-2-3, Jumbo, Nemadoro, Stevens e TOM-556. O programa obteve famílias com resistência múltipla a tospovírus, murcha de fusarium, geminivírus e nematóides das galhas e com características agronômicas superiores às cultivares Nemadoro e Malinta (Juliatti et al., 2001).

Em se tratando de tomate para processamento, torna-se necessária, além da avaliação do comportamento das famílias na fase de campo, a determinação do seu rendimento industrial. Desta forma, este trabalho foi conduzido para caracterizar e selecionar agronômica e industrialmente as melhores famílias provenientes deste programa, e comparar seus desempenhos com os híbridos mais utilizados pelos produtores na região de Patos de Minas, no sistema de produção com irrigação por gotejamento.

\section{MATERIAL E MÉTODOS}

Os ensaios foram conduzidos na Estação Experimental Fazenda Barreiro, pertencente a Unilever Bestfoods Brasil, situada no município de Patos de Minas (46 $51^{\circ}$ ' Oeste e $18^{\circ} 57^{\prime}$ Sul, 1100 $\mathrm{m}$ de altitude, temperatura média anual de $22,3{ }^{\circ} \mathrm{C}$ ).

Utilizaram-se 30 famílias de tomateiro, provenientes do Programa de Melhoramento do Tomateiro da UFU, obtidas por meio do método genealógico (Ramalho et al., 1993), e os híbridos comerciais Heinz 9553, Heinz 7155N2 e Hypeel 108, como testemunhas. O delineamento experimental adotado foi o de blocos casualizados, com 4 repetições. Foram usadas parcelas com linhas duplas de $10 \mathrm{~m}$ de comprimento, espaçadas $0,5 \mathrm{~m}$. O espaçamento entre linhas duplas foi de $1,3 \mathrm{~m}$ e a população foi de 27 mil plantas por hectare. A semeadura foi realizada em 19/05/01, em bandejas de polipropileno expandido. $\mathrm{O}$ transplantio para o campo ocorreu quando as mudas completaram 32 dias após a semeadura, com uma muda por cova. O solo da área experimental pertence à classe textural argiloso, possuindo $52,3 \%$ de argila, $23,6 \%$ de silte e $24,1 \%$ de areia, com densidades aparente e real de 1,0 e 2,7 respectivamente e fertilidade alta.

O solo foi preparado com uma aração a $25 \mathrm{~cm}$ de profundidade e gradeado. A seguir aplicou-se, em pré-plantio-incorporado, os herbicidas Trifluralina concentrado emulsionável $445 \mathrm{~g} / 1$ (1,3 L/ha) e Metribuzin suspensão concentrada $480 \mathrm{~g} / 1$ (0,9 L/ha). Na adubação de plantio utilizou-se de 1500 $\mathrm{kg} / \mathrm{ha}$ de $04-30-16+0,5 \% \mathrm{Zn}+0,02 \%$ B. No sulco de adubação aplicaram-se $30 \mathrm{~kg} / \mathrm{ha}$ de Carbofuran granulado 50 $\mathrm{g} / \mathrm{kg}$. A adubação de cobertura foi realizada com $80 \mathrm{~kg} / \mathrm{ha}$ de N, $62 \mathrm{~kg} / \mathrm{ha}$ de $\mathrm{P}_{2} \mathrm{O}_{5}$ e $100 \mathrm{~kg} /$ ha de $\mathrm{K}_{2} \mathrm{O}$ parcelados por meio de fertirrigação. A irrigação por gotejamento foi feita via subsuperfície utilizando-se fita Netafin ${ }^{\circledR} \operatorname{com} 120 \mathrm{~m}$ de comprimento, $1,65 \mathrm{~L} / \mathrm{h}$ de vazão por gotejador e $0,4 \mathrm{~m}$ de distância entre gotejadores, enterrada a $20 \mathrm{~cm}$ abaixo da superfície do solo. A umidade do solo foi monitorada por meio de tensiômetros instalados a 20 e a $40 \mathrm{~cm}$ de profundidade.

Procurou-se iniciar a aplicação das lâminas de irrigação sempre que a tensão de água no solo na profundidade de $20 \mathrm{~cm}$ alcançava 0,25-0,35 KPA. A evaporação real no período entre duas irrigações subsequentes foi determinada pela evaporação da água medida em tanque classe " $A$ " menos a precipitação pluviométrica no período. A lâmina real a ser aplicada foi calculada multiplicando-se a evaporação real por uma constante que variou conforme o estádio de desenvolvimento da cultura (Giordano et al., 2000). A partir de aproximadamente 100 dias do transplantio foi suspensa a irrigação no experimento. Mas, devido ao experimento ter sido conduzido no final da estação de cultivo (plantio tardio), a ocorrência de chuvas no final do ciclo limitou bastante o estresse hídrico que pretendia-se aplicar à cultura neste estádio. Os tratamentos fitossanitários foram feitos de acordo com o monitoramento das áreas experimentais, em função dos patógenos, pragas e plantas daninhas presentes. A colheita foi realizada aos 132 dias após o transplantio. 
Avaliou-se visualmente, em uma das repetições do ensaio, as características de hábito de crescimento (determinado ou indeterminado), porte (comprimento das hastes principais), uniformidade na parcela (cobertura foliar e porte de planta), formato do fruto (caqui, pêra, bloco, redondo, oval), firmeza (capacidade de resistência do fruto à compressão feita com a ponta dos dedos), cor externa [excelente (todos frutos da parcela com coloração vermelha)], preenchimento interno [frutos completos (ausência de vazios)], teor de água (obtido amassando-se as metades de três frutos por parcela com a palma das mãos), quantidade de sementes (através da escala comparativa variando de muito poucas a muitas sementes), retenção de pedúnculo [fenótipos “jointless" (ausência de joelho) e "jointed" (presença de joelho)], incidência de podridão apical e rachaduras longitudinais e/ou concêntricas

A produção total de frutos (PT) foi obtida pela soma das produções de frutos maduros, coloridos, verdes e podres. Considerou-se como produção de frutos comerciais (FC) aqueles frutos que se apresentaram maduros no dia da colheita. A porcentagem de frutos refugados para o processamento (FR) foi obtida pela soma das produções de frutos coloridos, verdes e podres dividida pela produção total. A partir de uma amostra de 20 frutos maduros coletados ao acaso dentro das parcelas foi obtido o peso médio dos frutos maduros (PMFM) no dia da colheita. Obteve-se o número de frutos maduros por planta (NFMP), fazendo-se a seguinte relação: $\mathrm{NFMP}=\mathrm{FC} /\left(\mathrm{PMFM}{ }^{*} \mathrm{NPH}\right)$, onde NPH é o número de plantas por hectare e FC (t/ha) é a produção de frutos maduros por hectare.

Para determinação das características industriais coletou-se, ao acaso, 20 frutos de tomate maduros, de cada parcela, no dia da colheita. As amostras foram imediatamente transportadas para o laboratório de tecnologia de alimentos na unidade industrial de processamento da Unilever Bestfoods Brasil, no município de Patos de Minas. Os frutos foram lavados e processados em liquidificador para obtenção do suco utilizado nas análises a seguir. A porcentagem de sólidos solúveis totais da amostra $\left({ }^{\circ}\right.$ Brix $)$ foi obtida em um refratômetro marca Bellingham+Stanley, modelo RFM320. Para determinar a acidez total titulável utilizou-se o equipamento marca Metrohm modelo 712S Titrino. Para a determinação do $\mathrm{pH}$ utilizou-se equipamento PerpHecT pH, marca Orion, modelo 310 com um eletrodo tipo Ross Sureflow, modelo 8172. A relação a/b é composta pelo valor de a, que mede a quantidade de vermelho (licopeno) presente na amostra quando positivo e pelo valor de b, que mede a quantidade de amarelo (beta-caroteno) presente na amostra quando positivo (Fonseca, 1982). Para obtenção da relação a/b (COR), utilizou-se colorímetro marca HunterLab, modelo ColorQUEST.

$O$ rendimento industrial de polpa foi obtido utilizando-se a fórmula: REND (t de polpa/ha) $=[(\mathrm{FC}(\mathrm{t} / \mathrm{ha}) \times 0,95) \times$ ${ }^{\circ}$ Brix do suco $] / 28$. Onde REND é o rendimento industrial de polpa concentrada a $28{ }^{\circ}$ Brix e FC (t/ha) é a produção de frutos maduros (Giordano et al., 2000).

As análises de variância [teste $\mathrm{F}$, modelo Yijk $=\mathrm{m}+\mathrm{Gi}+\mathrm{Bk}+$ Eik (Gomes, 1990)], comparações entre médias [teste de Scott-Knott (Scott \& Knott, 1974, Ferreira et al., 1999)], estimativas das variâncias fenotípicas, genotípicas e ambientais (Ramalho et al., 1993) e os coeficientes de correlação fenotípicos, genotípicos e ambientais (Cruz \& Ragazzi, 1997) foram processadas no Programa Computacional Genes, rotina blocos ao acaso com testemunhas adicionais (Cruz, 1997). A significância dos coeficientes de correlação fenotípica, genotípica e ambiental foram avaliadas pelo teste $\mathrm{t}$, em $5 \%$ de significância (Steel \& Torrie, 1980).

\section{RESULTADOS E DISCUSSÃO}

Na avaliação visual dos genótipos, os três híbridos comerciais obtiveram posição de destaque, pois mostraram-se adequados para o tipo industrial por apresentarem hábito de crescimento determinado, porte de planta pequeno, excelente uniformidade de plantas dentro da parcela, frutos firmes, com boa coloração, cheios ou bem preenchidos internamente, com teor de água exce- lente, quantidade de sementes normal, fenótipo "jointless" e ausência de podridão apical e rachaduras (Tabela 1). O mesmo ocorreu com as famílias UFU 06, UFU 15 e UFU 29. Porém, nestas famílias foi constatado porte de planta médio. As famílias UFU 29 e UFU 15 apresentaram cor externa dos frutos apenas regular, e a família UFU 29 apresentou também frutos com rachaduras e firmeza regular. Apesar de ter a maioria das características adequadas para o cultivo industrial, a família UFU 02 apresentou plantas com fenótipo "jointed", o que não é desejável. Segundo Giordano et al. (2000), a ausência de joelho no pedúnculo do fruto (fenótipo "jointless") faz com que este fique aderido à planta, facilitando a operação tanto de colheita manual como mecânica, e evitando o trabalho de remoção dos pedúnculos na linha de processamento. A família UFU 27 apresentou boas características visuais, porém tem formato de fruto tipo caqui. O formato de fruto similar aos frutos do grupo Santa Cruz (quadrado) são os preferidos para a indústria (Filgueira, 2000).

A análise de variância revelou diferenças significativas pelo teste $\mathrm{F}$ em $1 \%$ de probabilidade entre os genótipos e entre as famílias para todas as características avaliadas. $\mathrm{O}$ coeficiente de variação experimental (CV \%) foi inferior a $25 \%$ para todos os caracteres de campo e a $10 \%$ para os caracteres de laboratório, o que revela bom nível de precisão neste ensaio.

Os genótipos com maior PT foram: UFU 10, UFU 06, Heinz 7155 N2 e UFU 01 (Tabela 2).Para FC, os genótipos que se destacaram dos demais foram: Heinz 7155 N2, UFU 06, Heinz 9553 e Hypeel 108 (Tabela 2). Os genótipos com menor FR foram os híbridos Heinz 9553, Heinz 7155N2 e Hypeel 108 (Tabela 2), formando um grupo à parte dos demais. As melhores famílias, todas com desempenho inferior aos híbridos, foram: UFU 02, UFU 11, UFU 27, UFU 06, UFU 09 , UFU 25, UFU 24, UFU 29, UFU 15 e UFU 05, com valores variando de $14,96 \%$ a $20,70 \%$. Num experimento irrigado e com diversas variedades e híbridos, feito por três anos consecutivos em duas regiões da Espanha, Macua et al. (1999) também observaram a média 
Tabela 1. Características das plantas e dos frutos dos genótipos de tomateiro para processamento no sistema de produção irrigado via gotejamento. Patos de Minas, UFU, 2002.

\begin{tabular}{|c|c|c|c|c|c|c|c|c|c|c|c|c|}
\hline \multirow{2}{*}{ Genótipo } & \multicolumn{3}{|c|}{$\begin{array}{c}\text { Características da } \\
\text { Planta }\end{array}$} & \multicolumn{9}{|c|}{ Características do Fruto } \\
\hline & $\mathrm{HAB}^{3}$ & POR & UNI & FOR & FIR & CE & PRE & TA & QS & PA & PA & RA \\
\hline UFU 01 & $I^{1}$ & $G$ & $\mathrm{R}$ & $\mathrm{BI}$ & $\mathrm{R}$ & $B$ & $\mathrm{Ch}$ & $E$ & $P$ & Js & $\mathrm{Au}$ & $\mathrm{Au}$ \\
\hline UFU 02 & $\mathrm{D}$ & $\mathrm{Pq}$ & B & 0 & $\mathrm{~F}$ & $\mathrm{R}$ & $\mathrm{Ch}$ & E & $P$ & Jd & $\mathrm{Au}$ & $\mathrm{Au}$ \\
\hline UFU 03 & $\mathrm{D}$ & $G$ & $\mathrm{R}$ & $A$ & $\mathrm{R}$ & $\mathrm{R}$ & $\mathrm{N}$ & E & $N$ & Js & $\mathrm{Au}$ & $\mathrm{Au}$ \\
\hline UFU 04 & $\mathrm{D}$ & G & $\mathrm{R}$ & $\mathrm{Bl}$ & $\mathrm{R}$ & $\mathrm{B}$ & $\mathrm{N}$ & E & $N$ & Js & $\mathrm{Au}$ & $\mathrm{Au}$ \\
\hline UFU 05 & $\mathrm{D}$ & G & $E$ & $\mathrm{Rd}$ & $\mathrm{R}$ & $\mathrm{R}$ & $\mathrm{Ch}$ & $\mathrm{N}$ & $\mathrm{N}$ & $J d$ & $\mathrm{Au}$ & $\mathrm{Au}$ \\
\hline UFU 06 & $\mathrm{D}$ & $M$ & B & $\mathrm{Rd}$ & $\mathrm{F}$ & $B$ & $\mathrm{Ch}$ & $E$ & $N$ & Js & $\mathrm{Au}$ & $\mathrm{Au}$ \\
\hline UFU 07 & $\mathrm{D}$ & $G$ & $\mathrm{R}$ & $\mathrm{Ca}$ & $\mathrm{R}$ & $\mathrm{R}$ & $\mathrm{Ch}$ & $N$ & $N$ & $\mathrm{Jd}$ & $B x$ & At \\
\hline UFU 08 & $\mathrm{D}$ & $G$ & $P$ & $\mathrm{Rd} / \mathrm{Ca}^{2}$ & $\mathrm{R}$ & $B$ & $\mathrm{Ch}$ & $N$ & $N$ & $\mathrm{Jd} / \mathrm{Js}^{2}$ & $M$ & At \\
\hline UFU 09 & $\mathrm{D}$ & $M$ & $\mathrm{R}$ & $\mathrm{O} / \mathrm{BI}^{2}$ & $\mathrm{R}$ & $\mathrm{R}$ & Oc & $N$ & $P$ & $\mathrm{Jd}$ & $\mathrm{Au}$ & $\mathrm{Bx}$ \\
\hline UFU 10 & 1 & $G$ & $\mathrm{R}$ & $\mathrm{Rd}$ & $\mathrm{R}$ & $B$ & $\mathrm{Ch}$ & $N$ & $\mathrm{~N}$ & Js & $\mathrm{Au}$ & $\mathrm{Au}$ \\
\hline UFU 11 & $D$ & G & $B$ & $\mathrm{Rd} / \mathrm{Ca}^{2}$ & $\mathrm{R}$ & $\mathrm{R}$ & $\mathrm{Ch}$ & E & $N$ & Js & $\mathrm{Au}$ & $\mathrm{Au}$ \\
\hline UFU 12 & D & $G$ & $\mathrm{R}$ & $\mathrm{Rd} / \mathrm{Ca}^{2}$ & $\mathrm{R}$ & $B$ & $\mathrm{Ch}$ & $\mathrm{N}$ & $N$ & $\mathrm{Js} / \mathrm{Jd}^{2}$ & $\mathrm{Au}$ & $\mathrm{Bx}$ \\
\hline UFU 13 & D & $G$ & $\mathrm{R}$ & $\mathrm{Bl} / \mathrm{Pe}^{2}$ & $\mathrm{~F}$ & $B$ & $\mathrm{Ch}$ & E & $P$ & $\mathrm{Js} / \mathrm{Jd}^{2}$ & $\mathrm{Au}$ & $\mathrm{Au}$ \\
\hline UFU 14 & 1 & $G$ & $\mathrm{R}$ & $\mathrm{BI}$ & E & $B$ & Oc & E & $P$ & Js & $\mathrm{Au}$ & $\mathrm{Au}$ \\
\hline UFU 15 & D & $M$ & B & $\mathrm{Rd}$ & $\mathrm{F}$ & $\mathrm{R}$ & $\mathrm{Ch}$ & $\mathrm{N}$ & $N$ & Js & $\mathrm{Au}$ & $\mathrm{Au}$ \\
\hline UFU 16 & D & $G$ & $\mathrm{R}$ & $\mathrm{Ca} / \mathrm{Rd}^{2}$ & $\mathrm{R}$ & $\mathrm{R}$ & $\mathrm{Ch}$ & $N$ & $N$ & $\mathrm{Js} / \mathrm{Jd}^{2}$ & $\mathrm{Au}$ & At \\
\hline UFU 17 & 1 & G & $\mathrm{R}$ & $\mathrm{Rd} / \mathrm{Ca}^{2}$ & $\mathrm{~F}$ & $B$ & $\mathrm{Ch}$ & E & $N$ & $\mathrm{Jd}$ & $B x$ & M \\
\hline UFU 18 & $\mathrm{D}$ & $G$ & $\mathrm{R}$ & $\mathrm{Rd} / \mathrm{Ca}^{2}$ & $\mathrm{R}$ & $\mathrm{R}$ & $\mathrm{Ch}$ & $N$ & $N$ & $J d$ & $\mathrm{Au}$ & At \\
\hline UFU 19 & 1 & G & $\mathrm{R}$ & $\mathrm{Ca} / \mathrm{Rd}^{2}$ & $\mathrm{R}$ & $\mathrm{R}$ & $\mathrm{N}$ & $\mathrm{N}$ & $N$ & $\mathrm{Js} / \mathrm{Jd}^{2}$ & $\mathrm{M}$ & At \\
\hline UFU 20 & D & $G$ & $\mathrm{R}$ & $\mathrm{BI}$ & $\mathrm{R}$ & $\mathrm{R}$ & Oc & $E$ & $P$ & Js & $\mathrm{Au}$ & $\mathrm{Bx}$ \\
\hline UFU 21 & 1 & $G$ & $\mathrm{R}$ & $\mathrm{Ca} / \mathrm{Rd}^{2}$ & $\mathrm{~F}$ & $\mathrm{R}$ & $\mathrm{Ch}$ & $N$ & $N$ & $J d$ & $B x$ & At \\
\hline UFU 22 & $\mathrm{D}$ & $G$ & $\mathrm{R}$ & $\mathrm{Rd}$ & $\mathrm{F}$ & $\mathrm{R}$ & $\mathrm{Ch}$ & $N$ & $\mathrm{~N}$ & Js & $\mathrm{Au}$ & $\mathrm{Au}$ \\
\hline UFU 23 & 1 & $G$ & $\mathrm{R}$ & $\mathrm{A} / \mathrm{Pe}^{2}$ & $\mathrm{~F}$ & $\mathrm{R}$ & Oc & $E$ & $P$ & Js & $\mathrm{Au}$ & $\mathrm{Au}$ \\
\hline UFU 24 & 1 & $G$ & $\mathrm{R}$ & $\mathrm{Rd} / \mathrm{BI}^{2}$ & $\mathrm{R}$ & $\mathrm{R}$ & $\mathrm{N}$ & $E$ & $P$ & Js & $\mathrm{Au}$ & $\mathrm{Au}$ \\
\hline UFU 25 & $D$ & G & $\mathrm{R}$ & $\mathrm{Rd}$ & $\mathrm{F}$ & $B$ & $\mathrm{Ch}$ & $N$ & $N$ & Js & $\mathrm{Au}$ & $B x$ \\
\hline UFU 26 & $\mathrm{D}$ & $M$ & B & $\mathrm{Pe}$ & $\mathrm{F}$ & $B$ & Oc & MS & MP & Js & $\mathrm{Au}$ & $\mathrm{Au}$ \\
\hline UFU 27 & $\mathrm{D}$ & $M$ & B & $\mathrm{Ca}$ & $\mathrm{R}$ & $\mathrm{R}$ & $\mathrm{Ch}$ & $N$ & $N$ & Js & $\mathrm{Au}$ & $\mathrm{Au}$ \\
\hline UFU 28 & 1 & $G$ & $\mathrm{R}$ & $\mathrm{Rd}$ & $\mathrm{R}$ & $B$ & $\mathrm{Ch}$ & $\mathrm{N}$ & $N$ & Js & $\mathrm{Au}$ & $\mathrm{Bx}$ \\
\hline UFU 29 & $\mathrm{D}$ & $M$ & B & $\mathrm{Rd}$ & $\mathrm{R}$ & $\mathrm{R}$ & $\mathrm{Ch}$ & $E$ & $\mathrm{~N}$ & Js & $\mathrm{Au}$ & M \\
\hline UFU 30 & 1 & $G$ & $\mathrm{R}$ & $\mathrm{Rd}$ & $\mathrm{F}$ & $B$ & $\mathrm{Ch}$ & E & $N$ & $\mathrm{Jd}$ & $\mathrm{Au}$ & $B x$ \\
\hline Heinz 9553 & $\mathrm{D}$ & $\mathrm{Pq}$ & $E$ & $A$ & $\mathrm{~F}$ & $\mathrm{~B}$ & $\mathrm{Ch}$ & E & $N$ & Js & $\mathrm{Au}$ & $\mathrm{Au}$ \\
\hline Heinz 7155N2 & $\mathrm{D}$ & $\mathrm{Pq}$ & $E$ & $A$ & $\mathrm{~F}$ & $\mathrm{R}$ & $\mathrm{Ch}$ & E & $N$ & Js & $\mathrm{Au}$ & $\mathrm{Au}$ \\
\hline Hypeel 108 & $\mathrm{D}$ & $\mathrm{Pq}$ & B & $A$ & $\mathrm{R}$ & $\mathrm{B}$ & $\mathrm{Ch}$ & $\mathrm{N}$ & $\mathrm{N}$ & Js & $\mathrm{Au}$ & $\mathrm{Au}$ \\
\hline
\end{tabular}

${ }^{1}$ I (Indeterminado); D (determinado); G (grande); M (médio); Pq (pequeno); C (comPActo); E (excelente); B (bom); R (regular); Ru (ruim); Bl (bloco); O (oval); A (alongado); Rd (redondo); Ca (caqui); Pe (pêra); F (firme); N (normal); Ch (cheio); Oc (ocado); MS (muito seco); P (poucas); MP (muito poucas); Js (jointless); Jd (jointed); Au (ausente); Bx (baixa); At (alta)

${ }^{2}$ Plantas segregando para a característica dentro da parcela

${ }^{3}$ Características da Planta: Hábito de crescimento (HAB), Porte (POR), Uniformidade (UNI); Características do Fruto: Formato do fruto (FOR), Firmeza do fruto (FIR), Cor Externa (CE), Preenchimento interno (PRE), Teor de Água (TA), Quantidade de Sementes (QS), Facilidade de Colheita (PA), Podridão Apical (PA), Rachaduras (RA).

de $20 \%$ de frutos inadequados, o que coloca estas famílias dentro do padrão aceitável para a tomaticultura industrial.
Existe uma tendência de se preferir, para a indústria, genótipos que produzam frutos menores, com peso médio variando entre 50 e $100 \mathrm{~g}$, com formato próximo ao do grupo Santa Cruz, que conferem maior resistência ao transporte 
Tabela 2. Médias dos genótipos para produção total (PT, t/ha), produção de frutos adequados ao processamento (FC, $t / h a)$, produção de frutos inadequados ao processamento (FR), peso médio do fruto maduro (PMFM, g), número de frutos maduros por planta (NFMP). Patos de Minas, UFU, 2002.

\begin{tabular}{|c|c|c|c|c|c|c|}
\hline \multirow{2}{*}{$\begin{array}{l}\text { Genótipo } \\
\text { UFU } 01\end{array}$} & \multirow{2}{*}{$\begin{array}{r}\mathbf{P T} \\
133,2 a\end{array}$} & FC & FR & PMFM & \multicolumn{2}{|c|}{ NFMP } \\
\hline & & $98,8 \quad b$ & $26,0 \quad c$ & 221,3 & 16,6 & e \\
\hline UFU 02 & $116,3 \mathrm{~b}$ & $99,5 \mathrm{~b}$ & $15,0 \mathrm{~b}$ & $116,9 b$ & 30,0 & $c$ \\
\hline UFU 03 & $93,6 \quad c$ & $66,4 \quad c$ & 29,2 & $145,6 \quad c$ & 16,4 & $\mathrm{e}$ \\
\hline UFU 04 & $105,3 \quad c$ & $79,6 \quad c$ & $24,3 \quad c$ & $211,3 \quad d$ & 14,7 & $\mathrm{e}$ \\
\hline UFU 05 & $107,2 \quad c$ & $84,8 \quad c$ & 20,7 b & $145,0 \quad c$ & 20,8 & $d$ \\
\hline UFU 06 & $138,1 a$ & $114,7 a$ & 17,2 b & $116,9 \mathrm{~b}$ & 35,7 & b \\
\hline UFU 07 & $107,7 \quad c$ & $78,6 \quad c$ & $27,0 \quad d$ & $223,1 \quad d$ & 15,2 & e \\
\hline UFU 08 & $108,4 \quad c$ & $85,0 \quad c$ & $21,5 \quad c$ & $180,0 \quad c$ & 18,2 & $d$ \\
\hline UFU 09 & $96,5 \quad c$ & $78,4 \quad c$ & 18,9 b & $128,8 b$ & 20,3 & $d$ \\
\hline UFU 10 & $140,9 a$ & $99,5 \mathrm{~b}$ & $29,0 \quad d$ & $126,3 \mathrm{~b}$ & 28,4 & $c$ \\
\hline UFU 11 & $110,3 \quad c$ & $92,2 \mathrm{~b}$ & $16,3 \mathrm{~b}$ & $155,0 \quad c$ & 22,8 & $d$ \\
\hline UFU 12 & $123,0 \mathrm{~b}$ & 96,8 b & $21,7 \quad c$ & $134,4 \mathrm{~b}$ & 25,8 & c \\
\hline UFU 13 & $122,6 b$ & 91,4 b & $25,0 \quad c$ & $143,8 \quad c$ & 23,4 & $d$ \\
\hline UFU 14 & $109,8 \quad c$ & $80,9 \quad c$ & 26,8 & $156,3 \quad c$ & 19,6 & d \\
\hline UFU 15 & 111,3 & $89,1 \quad c$ & $20,1 b$ & $160,0 \quad c$ & 20,4 & $d$ \\
\hline UFU 16 & $85,0 \quad c$ & $63,1 \quad c$ & $25,6 \quad c$ & $154,4 \quad c$ & 15,2 & e \\
\hline UFU 17 & $99,0 \quad c$ & 77,7 & $21,4 \quad c$ & $161,3 \quad c$ & 17,6 & e \\
\hline UFU 18 & $100,8 \quad c$ & 78,4 & $22,2 \quad c$ & $150,0 \quad c$ & 18,6 & $d$ \\
\hline UFU 19 & $100,5 \quad c$ & 76,4 & $24,0 \quad c$ & $163,8 \quad c$ & 16,7 & e \\
\hline UFU 20 & $110,8 \quad c$ & $85,1 \quad c$ & $22,9 \quad c$ & 207,5 & 15,2 & e \\
\hline UFU 21 & $102,3 \quad c$ & 80,0 & $22,4 \quad c$ & $165,8 \quad c$ & 17,6 & $\mathrm{e}$ \\
\hline UFU 22 & $114,1 b$ & 89,7 & $21,4 \quad c$ & $163,8 \quad c$ & 21,4 & $d$ \\
\hline UFU 23 & $110,0 \quad c$ & 74,7 & $32,3 \quad d$ & $129,4 \mathrm{~b}$ & 21,6 & $d$ \\
\hline UFU 24 & $110,1 \quad c$ & 88,9 & 19,2 b & $133,1 \quad b$ & 25,0 & C \\
\hline UFU 25 & $107,2 \quad c$ & 86,4 & $19,1 \mathrm{~b}$ & $156,9 \quad c$ & 21,9 & $d$ \\
\hline UFU 26 & $115,2 b$ & $87,8 \quad c$ & $23,7 \quad c$ & $168,1 \quad c$ & 18,8 & $d$ \\
\hline UFU 27 & $95,6 \quad c$ & 79,4 & $17,0 \mathrm{~b}$ & $148,8 \quad c$ & 19,9 & $d$ \\
\hline UFU 28 & $103,2 \quad c$ & $73,2 \quad c$ & 29,1 & $174,4 \quad \mathrm{c}$ & 15,8 & $\mathrm{e}$ \\
\hline UFU 29 & $95,1 \quad \mathrm{c}$ & $77,2 \quad c$ & 19,6 b & $158,8 \quad c$ & 19,1 & $d$ \\
\hline UFU 30 & $108,5 \quad c$ & $74,4 \quad c$ & 31,4 & $98,1 \mathrm{a}$ & 28,9 & C \\
\hline Heinz 7155 N2 & $136,5 a$ & $122,8 \mathrm{a}$ & $10,2 a$ & $119,4 b$ & 38,2 & $b$ \\
\hline Heinz 9553 & $124,0 \mathrm{~b}$ & $112,9 a$ & $8,8 a$ & $82,5 a$ & $50,2 a$ & \\
\hline Hypeel 108 & $117,3 \mathrm{~b}$ & $102,4 \mathrm{~b}$ & $12,8 a$ & $139,4 \mathrm{~b}$ & 26,9 & $\mathrm{c}$ \\
\hline Médias & 110,90 & 86,85 & 21,87 & 154,37 & 22,33 & \\
\hline
\end{tabular}

Letras iguais, nas colunas, indicam médias iguais pelo teste de agrupamento de médias de Scott-Knott, ao nível de 5\% de probabilidade.

(Filgueira, 2000). Mas, de acordo com Giordano et al. (2000), o fruto não deve possuir diâmetro inferior a $3 \mathrm{~cm}$, o que acarretaria diminuição do rendimento no processo de colheita. Resende \& Costa (2000) obtiveram frutos do híbrido Hypeel 108 com 101,54 g/fruto, mas observaram que as cultivares mais produtivas produziram frutos com baixo peso médio e maior número de frutos por planta. Prieto et al. (1999) obtiveram valores para peso médio de frutos menores com irrigação por gotejamento, quando comparados aos valores para irrigação por aspersão, mas essa diferença foi compensada pelo maior número de frutos sob irrigação por gotejamento. Então, decidiu-se selecionar como melhores para PMFM, os genótipos
Heinz 9553, UFU 30, UFU 06, UFU 02, Heinz 7155N2, UFU 09, UFU 10, UFU 23, UFU 24, UFU 12 e Hypeel 108, com pesos médios de fruto variando de 82,50 g a 139,38 g (Tabela 2), por apresentarem os frutos com menores pesos médios.

O híbrido Heinz 9553 mostrou NMFP superior aos demais genótipos, sendo seguido pelo híbrido Heinz 
Tabela 3. Médias dos genótipos para coloração do suco (Cor), ${ }^{o b r i x ~(\% ~ s o ́ l i d o s ~ s o l u ́ v e i s ~ t o t a i s), ~ p H, ~ a c i d e z ~ t o t a l ~ t i t u l a ́ v e l ~(m g ~ a ́ c . ~ c i ́ t r i c o / ~}$ $100 \mathrm{~g}$ suco) e rendimento industrial (t polpa $28^{\circ} \mathrm{brix} / \mathrm{ha}$ ). Patos de Minas, UFU, 2002.

\begin{tabular}{|c|c|c|c|c|c|c|c|c|c|}
\hline \multirow{2}{*}{$\begin{array}{l}\text { Genótipo } \\
\text { UFU } 01\end{array}$} & \multirow{2}{*}{\multicolumn{2}{|c|}{$\frac{\text { Cor }}{2,52 a^{3}}$}} & \multicolumn{2}{|c|}{${ }^{\circ}$ Brix } & pH & \multicolumn{2}{|l|}{ Acidez } & \multicolumn{2}{|c|}{ Rendimento } \\
\hline & & & $4,94 a$ & & $4,54 \quad c$ & $257,5^{2}$ & $\mathrm{c}$ & $16,61 \mathrm{a}$ & \\
\hline UFU 02 & 2,27 & $b$ & 4,00 & $d$ & $4,45^{1} b$ & $285,0^{2}$ & & 13,51 & $b$ \\
\hline UFU 03 & $2,32 a$ & & 4,48 & b & $4,41 a$ & $300,0^{2} \mathrm{~b}$ & b & 10,04 & $b$ \\
\hline UFU 04 & $2,45 a$ & & $4,91 a$ & & $4,60^{1}$ & $250,0^{2}$ & $c$ & 13,27 & $b$ \\
\hline UFU 05 & $2,44 a$ & & 4,39 & c & $4,48 \mathrm{~b}$ & $275,0^{2}$ & & 12,70 & $b$ \\
\hline UFU 06 & $2,41 a$ & & 4,22 & c & $4,53 \quad c$ & $277,5^{2}$ & & $16,40 \mathrm{a}$ & \\
\hline UFU 07 & 2,17 & $b$ & $4,94 a$ & & $4,48 \mathrm{~b}$ & $315,0^{2} a$ & & 13,17 & $b$ \\
\hline UFU 08 & 2,23 & $b$ & 4,65 & $b$ & $4,47 \mathrm{~b}$ & $327,5^{2} a$ & & 13,41 & $b$ \\
\hline UFU 09 & $2,35 a$ & & 4,36 & $c$ & $4,36^{1} a$ & $320,0^{2} a$ & & 11,62 & $b$ \\
\hline UFU 10 & $2,34 a$ & & $4,83 a$ & & $4,45 \mathrm{~b}$ & $297,5^{2} b$ & & $16,28 \mathrm{a}$ & \\
\hline UFU 11 & 2,24 & $b$ & 4,17 & $c$ & $4,46 \mathrm{~b}$ & $300,0^{2} \mathrm{~b}$ & & 13,07 & $b$ \\
\hline UFU 12 & 2,19 & $b$ & $4,75 a$ & & $4,41^{1} a$ & $335,0 a$ & & $15,63 a$ & \\
\hline UFU 13 & 2,19 & $b$ & 4,58 & $b$ & $4,44 \mathrm{~b}$ & $292,5^{2}$ & & $14,21 \mathrm{a}$ & \\
\hline UFU 14 & 2,23 & $b$ & 4,34 & c & $4,51 \quad \mathrm{c}$ & $282,5 \mathrm{~b}$ & & 11,90 & $b$ \\
\hline UFU 15 & $2,30 a$ & & 4,14 & c & $4,42 \mathrm{~b}$ & $317,5^{2} a$ & & 12,54 & $b$ \\
\hline UFU 16 & 2,28 & $b$ & $5,01 \mathrm{a}$ & & $4,36^{1} a$ & $367,5^{2} a$ & & 10,76 & $b$ \\
\hline UFU 17 & 2,22 & $b$ & $4,81 a$ & & $4,45 \mathrm{~b}$ & $332,5 a$ & & 12,69 & $b$ \\
\hline UFU 18 & 2,02 & c & 4,51 & b & $4,36^{1} a$ & $337,5 a$ & & 11,99 & $b$ \\
\hline UFU 19 & 2,11 & c & 4,43 & c & $4,34^{1} a$ & $325,0 a$ & & 11,51 & $b$ \\
\hline UFU 20 & $2,52 a$ & & $4,75 a$ & & $4,62^{1} \quad d$ & $245,0^{2}$ & c & 13,72 & $b$ \\
\hline UFU 21 & 2,17 & $b$ & 4,31 & c & $4,36^{1} a$ & $346,7 a$ & & 11,75 & $b$ \\
\hline UFU 22 & $2,31 a$ & & 4,27 & c & $4,36^{1} a$ & $345,0 a$ & & 13,00 & $b$ \\
\hline UFU 23 & 2,27 & $b$ & 4,45 & $c$ & $4,50 \quad c$ & $247,5^{2}$ & c & 11,27 & $b$ \\
\hline UFU 24 & $2,41 a$ & & $4,73 a$ & & $4,47 \mathrm{~b}$ & $312,5^{2} a$ & & $14,28 a$ & \\
\hline UFU 25 & 2,27 & $b$ & 4,55 & $b$ & $4,54 \quad c$ & $280,0^{2}$ & & 13,33 & $b$ \\
\hline UFU 26 & 2,07 & c & 3,76 & $d$ & $4,45^{1} \quad b$ & $277,5^{2}$ & $b$ & 11,17 & $b$ \\
\hline UFU 27 & 2,15 & $b$ & 4,22 & c & $4,42 a$ & $330,0 a$ & & 11,38 & $b$ \\
\hline UFU 28 & $2,40 a$ & & $4,84 a$ & & $4,54 \quad c$ & $280,0^{2} b$ & b & 12,01 & $b$ \\
\hline UFU 29 & 2,19 & $\mathrm{~b}$ & 4,19 & c & $4,39^{1} a$ & $342,5 a$ & & 10,83 & $b$ \\
\hline UFU 30 & 2,06 & c & $4,78 a$ & & $4,39^{1} a$ & $332,5 a$ & & 12,07 & $b$ \\
\hline Heinz 7155N2 & 2,20 & $\mathrm{~b}$ & 3,78 & $d$ & $4,47 \mathrm{~b}$ & $286,7^{2} \mathrm{~b}$ & & $15,74 a$ & \\
\hline Heinz 9553 & $2,38 a$ & & 3,92 & $d$ & $4,44 \mathrm{~b}$ & $305,0^{2} a$ & & $15,04 a$ & \\
\hline Hypeel 108 & 2,18 & $\mathrm{~b}$ & 4,14 & $\mathrm{~d}$ & $4,63^{1}$ & $255,0^{2}$ & c & $14,37 \mathrm{a}$ & \\
\hline Médias & 2,27 & & 4,46 & & 4,46 & 302,5 & & 13,07 & \\
\hline
\end{tabular}

${ }^{1} \mathrm{e}^{2}$ indicam médias diferentes de 4,5 e de $350 \mathrm{mg}$ de ácido cítrico/100g, respectivamente, pelo teste $\mathrm{t}$ ao nível de $5 \%$ de probabilidade;

${ }^{3}$ Letras iguais, nas colunas, indicam médias iguais pelo teste de agrupamento de médias de Scott-Knott, ao nível de 5\% de probabilidade.

$7155 \mathrm{~N} 2$ e pela família UFU 06, num segundo grupo (Tabela 2).

Segundo Conceição (1981), para que o suco de tomate seja classificado no mais alto grau, deve apresentar porcentagem de vermelho acima de $65 \%$. Porcentagens de vermelho abaixo de $53 \%$ indicam tomate abaixo do padrão. Os genótipos com maior valor para relação a/b (COR) foram: UFU 20, UFU 01, UFU 04, UFU 05, UFU 24, UFU 06, UFU 28, Heinz 9553, UFU 09, UFU 10, UFU 03 e UFU 22 (Tabela 3). Os genótipos agrupados pelo teste de Scott-Knott na letra "b" também apresentaram valores da COR adequados para o processamento.
Os genótipos que apresentaram maiores médias para ${ }^{\circ}$ Brix (Tabela 3 ) foram: UFU 16, UFU 01, UFU 07, UFU 04, UFU 28, UFU 10, UFU 17, UFU 30, UFU 20, UFU 12 e UFU 24. Segundo Silva et al. (1994), a matéria-prima recebida pelas indústrias no Brasil tem tido baixa porcentagem de sólidos so- 
Tabela 4. Médias das famílias, variâncias fenotípica média $\left(\sigma_{\mathrm{F}}^{-2}\right)$, genotípica média $\left(\sigma_{\mathrm{G}}^{-2}\right)$ e ambiental média $\left(\sigma_{\mathrm{A}}^{-2}\right)$ e herdabilidade baseada na média de família $\left(h^{2}\right)$ das famílias de tomateiro. Patos de Minas, UFU, 2002.

\begin{tabular}{lccccc}
\hline & Rend. $^{\mathbf{1}}$ & PT & FC & FR & PMFM \\
\hline Médias & 12,87 & 109,39 & 84,27 & 23,00 & 158,31 \\
$\mathrm{~s}^{2} \mathrm{~F}$ & 2,8833 & 160,0369 & 116,4069 & 20,1272 & 747,8086 \\
$\mathrm{~s}^{2} \mathrm{G}$ & 1,9081 & 114,6894 & 78,5824 & 16,0224 & 602,0292 \\
$\mathrm{~s}^{2} \mathrm{~A}$ & 0,9752 & 45,3476 & 37,8248 & 4,1049 & 145,7794 \\
\hline $\mathrm{h}^{2}$ & 66,18 & 71,66 & 67,51 & 79,61 & 80,51 \\
\hline & NFMP & Cor & ${ }^{\circ}$ Brix & pH & Acid. \\
\hline Médias & 20,72 & 2,27 & 4,51 & 4,45 & 302,45 \\
$\mathrm{~s}^{2} \mathrm{~F}$ & 25,3689 & 0,0169 & 0,0987 & 0,0051 & 1075,7440 \\
$\mathrm{~s}^{2} \mathrm{G}$ & 20,2345 & 0,0140 & 0,0877 & 0,0046 & 932,5712 \\
$\mathrm{~s}^{2} \mathrm{~A}$ & 5,1345 & 0,0028 & 0,0109 & 0,0006 & 143,1728 \\
\hline $\mathrm{h}^{2}$ & 79,76 & 83,11 & 88,86 & 89,28 & 86,69 \\
\hline
\end{tabular}

${ }^{1}$ Rend.: rendimento industrial (t polpa $28^{\circ}$ brix/ha), PT: produção total (kg/ha), PA: produção de frutos adequados ao processamento (kg/ha), PI: produção de frutos inadequados ao processamento (\%), PMFM: peso médio do fruto maduro (g), NFMP: número de frutos maduros por planta, Cor: coloração do suco (a/b), ${ }^{\circ}$ Brix: \% sólidos solúveis totais, e Acid.: acidez total titulável (mg ác. cítrico/100g suco).

lúveis totais $\left(4,5^{\circ} \mathrm{Brix}\right)$, apesar de existirem cultivares que apresentaram valores próximos de 6,0. Em experimento realizado com 28 genótipos comerciais, dentre os quais estavam os híbridos Hypeel 108 (3,58 Brix) e Heinz 9553 (3,14 ${ }^{\circ}$ Brix), Cintra et al. (2000) não observaram valores médios de sólidos solúveis totais superiores a $4,0^{\circ}$ Brix, o que poderia estar relacionado com o período chuvoso ocorrido por ocasião das colheitas. Já Resende \& Costa (2000) observaram que a cultivar de polinização aberta IPA-5 apresentou o mais baixo brix $\left(4,3^{\circ} \mathrm{Brix}\right)$ apesar de ter sido a mais produtiva do ensaio, enquanto que o híbrido Hypeel 108, um dos genótipos menos produtivos, apresentou concentração de sólidos solúveis de $5,4^{\circ}$ Brix. Por outro lado, Cerne \& Resnik (1994) constataram maior ${ }^{\circ}$ Brix médio para as cultivares de polinização aberta $\left(4,6^{\circ}\right.$ Brix $)$ em relação às cultivares híbridas $\left(4,1^{\circ}\right.$ Brix $)$.

Indiretamente, o $\mathrm{pH}$ pode afetar a cor do produto final, pois se muito alto, exigirá maiores temperaturas e tempo para a esterilização, especialmente para a eliminação do microorganismo Clostridium botulinum (Giordano et al., 2000). A FDA Regulations on Thermal Processing of Food aponta o $\mathrm{pH}=4,6$ como o máximo permitido, para o suco do tomate fresco com destinação industrial. No presente ensaio, as famílias UFU 04 e UFU 20 e o híbrido Hypeel
108 (Tabela 3) foram os únicos genótipos a mostrarem valores de $\mathrm{pH}$ acima do máximo recomendado.

A acidez total titulável também influencia o sabor, pois mede a quantidade de ácidos orgânicos e indica a adstringência do fruto. Além disso, frutos apresentando valores de ácido cítrico abaixo de $350 \mathrm{mg} / 100 \mathrm{~g}$ de peso fresco requerem aumento no tempo e na temperatura de processamento, para evitar a proliferação de microorganismos nos produtos processados (Giordano et al., 2000). Entre todos, 22 genótipos apresentaram valores para ACID menores que $350 \mathrm{mg} / 100 \mathrm{~g}$ (Tabela 3). Entre esses genótipos estavam os híbridos Hypeel 108, Heinz 9553 e Heinz 7155 N2 e as famílias UFU 06, UFU 02 e UFU 15. Cintra et al. (2000), em experimento realizado em Jaboticabal, de junho a dezembro/99, com 28 genótipos comerciais de tomate para processamento (híbridos e linhagens), também não encontraram nenhum genótipo com acidez superior a $0,35 \%$ de ácido cítrico no suco.

Para REND que, segundo Conceição (1981), é determinado em função do conjunto das características agronômicas e industriais das cultivares, os melhores genótipos foram: UFU 01 , UFU 06, UFU 10, Heinz 7155N2, UFU 12, Heinz 9553, Hypeel 108, UFU 24 e UFU 13 (Tabela 3).
Os caracteres avaliados que apresentaram maiores variâncias fenotípicas foram PT, FC, PMFM, e ACID. As variâncias genotípicas foram análogas às superiores fenotípicas para todos os caracteres (Tabela 4).

Com exceção do ${ }^{\circ}$ Brix e do NFMP, a variável ACID apresentou correlações fenotípica e genotípica negativas com as demais características avaliadas (Tabela 5). Desta forma o aumento da acidez não pode ser buscado indiretamente por meio da seleção para outras características, sendo favorecido, entretanto, pelas mesmas condições ambientais que promovem aumento na COR, como revela a existência de correlação ambiental positiva entre as duas variáveis. Mas, a ocorrência de altas variâncias fenotípica e genotípica (Tabela 4) indicam a possibilidade de bons ganhos genéticos entre as famílias, para esta variável.

$\mathrm{O}{ }^{\circ}$ Brix apresentou correlação fenotípica e genotípica positiva com REND, PI, COR, PMFM e Ph e negativa com PT (somente fenotípica), FC e NFMP. Estes valores revelam uma pequena tendência de diminuição da porcentagem de sólidos solúveis com o aumento da produtividade do tomateiro. Assim, torna-se mais complicada a seleção de materiais que atendam de forma superior as duas características.

Para Conceição (1981), as porcentagens de sólidos solúveis totais podem 
Tabela 5. Estimativa dos coeficientes de correlação fenotípica (acima da diagonal), genotípica (acima da diagonal, entre parênteses) e de ambiente (abaixo da diagonal) entre variáveis do tomateiro. Patos de Minas, UFU, 2002.

\begin{tabular}{|c|c|c|c|c|c|c|c|c|c|c|}
\hline & Rend $^{1}$ & PT & PA & PI & ${ }^{\circ}$ Brix & Cor & PMFM & NFMP & $\mathrm{pH}$ & Acid \\
\hline Rend & & $\begin{array}{c}0,8711^{*} \\
(0,8795)^{*}\end{array}$ & $\begin{array}{c}0,846^{*} \\
(0,7946)^{\star}\end{array}$ & $\begin{array}{c}-0,1583 \\
(-0,0242)\end{array}$ & $\begin{array}{l}0,2979^{*} \\
(0,282)^{\star}\end{array}$ & $\begin{array}{c}0,4399^{\star} \\
(0,5154)^{\star}\end{array}$ & $\begin{array}{c}0,0442 \\
(0,0145)\end{array}$ & $\begin{array}{c}0,5027^{*} \\
(0,4617)^{\star}\end{array}$ & $\begin{array}{c}0,4358^{*} \\
(0,5435)^{\star}\end{array}$ & $\begin{array}{c}-0,3084^{*} \\
(-0,3906)^{*}\end{array}$ \\
\hline PT & $0,8574^{*}$ & & $\begin{array}{c}0,8819^{*} \\
(0,8652)^{*}\end{array}$ & $\begin{array}{c}0,0213 \\
(0,0767)\end{array}$ & $\begin{array}{l}-0,0053^{*} \\
(-0,0163)\end{array}$ & $\begin{array}{c}0,2856^{*} \\
(0,2904)^{*}\end{array}$ & $\begin{array}{c}-0,1001 \\
(-0,1553)\end{array}$ & $\begin{array}{c}0,6157^{*} \\
(0,6136)^{*}\end{array}$ & $\begin{array}{c}0,3808^{*} \\
(0,4488)^{*}\end{array}$ & $\begin{array}{c}-0,4364^{*} \\
(-0,5449)^{*}\end{array}$ \\
\hline PA & $0,9499^{*}$ & $0,9231^{*}$ & & $\begin{array}{c}-0,4479^{*} \\
(-0,4315)^{*}\end{array}$ & $\begin{array}{c}-0,2521^{*} \\
(-0,3573)^{*}\end{array}$ & $\begin{array}{c}0,2804^{*} \\
(0,3093)^{*}\end{array}$ & $\begin{array}{c}-0,1356 \\
(-0,2168)^{\star}\end{array}$ & $\begin{array}{c}0,6857^{*} \\
(0,6959)^{*}\end{array}$ & $\begin{array}{c}0,2999^{*} \\
(0,3565)^{*}\end{array}$ & $\begin{array}{c}-0,3098^{*} \\
(-0,3872)^{*}\end{array}$ \\
\hline $\mathrm{PI}$ & $-0,5358^{*}$ & $-0,1523$ & $-0,5111^{*}$ & & $\begin{array}{c}0,5045^{\star} \\
(0,6442)^{*}\end{array}$ & $\begin{array}{c}-0,0501 \\
(-0,0789)\end{array}$ & $\begin{array}{c}0,0776 \\
(0,1181)\end{array}$ & $\begin{array}{c}-0,2628^{*} \\
(-0,2486)^{*}\end{array}$ & $\begin{array}{c}0,0916 \\
(0,1218)\end{array}$ & $\begin{array}{l}-0,1770^{*} \\
(-0,239)^{*}\end{array}$ \\
\hline${ }^{\circ}$ Brix & $0,4208^{*}$ & 0,0432 & 0,1296 & $-0,2478^{*}$ & & $\begin{array}{c}0,3018^{*} \\
(0,33)^{*}\end{array}$ & $\begin{array}{c}0,2983^{*} \\
(0,3225)^{\star}\end{array}$ & $\begin{array}{l}-0,2838^{*} \\
(-0,345)^{*}\end{array}$ & $\begin{array}{c}0,2718^{*} \\
(0,3085)^{*}\end{array}$ & $\begin{array}{c}0,0000 \\
(-0,0002)\end{array}$ \\
\hline Cor & $0,2411^{*}$ & $0,281^{*}$ & $0,2081^{*}$ & 0,076 & 0,1327 & & $\begin{array}{c}0,3186^{*} \\
(0,4207)^{\star}\end{array}$ & $\begin{array}{c}-0,0336 \\
(-0,1039)\end{array}$ & $\begin{array}{c}0,6465^{*} \\
(0,7524)^{*}\end{array}$ & $\begin{array}{c}-0,5725^{*} \\
(-0,7153)^{\star}\end{array}$ \\
\hline PMFM & 0,1308 & 0,0762 & 0,0964 & $-0,085$ & $0,1734^{*}$ & $-0,1407$ & & $\begin{array}{c}-0,7855^{*} \\
(-0,8365)^{*}\end{array}$ & $\begin{array}{c}0,3889^{*} \\
(0,4188)^{*}\end{array}$ & $\begin{array}{c}-0,2566^{*} \\
(-0,2809)^{*}\end{array}$ \\
\hline NFMP & $0,6394^{*}$ & $0,634^{*}$ & $0,6824^{*}$ & $-0,3184^{*}$ & 0,0449 & $0,2758^{*}$ & $-0,5797^{*}$ & & $\begin{array}{c}-0,043 \\
(-0,0459)\end{array}$ & $\begin{array}{c}-0,0374 \\
(-0,0517)\end{array}$ \\
\hline $\mathrm{pH}$ & 0,0947 & 0,1253 & 0,1239 & $-0,0752$ & $-0,0274$ & $-0,0124$ & $0,2342^{*}$ & $-0,0289$ & & $\begin{array}{c}-0,8526^{*} \\
(-0,9244)^{*}\end{array}$ \\
\hline Acid & $-0,0592$ & $-0,0357$ & $-0,0655$ & 0,1309 & 0,0015 & $0,2315^{*}$ & $-0,1363$ & 0,0344 & $-0,3292^{*}$ & \\
\hline
\end{tabular}

${ }^{1}$ Rendimento industrial (Rend), Produção total (PT), Produção de frutos adequados ao processamento (FC), Porcentagem de frutos inadequados ao processamento (FR), \% sólidos solúveis totais ( ${ }^{\circ}$ Brix), Cor do suco (Cor), Peso médio do fruto maduro (PMFM), Número de frutos maduros por planta (NFMP), $\mathrm{pH}$ e Acidez (Acid)

* indica significância pelo teste $t$ ao nível de $5 \%$ de probabilidade.

sofrer grandes variações de acordo com cultivares, características do solo e especialmente pelas chuvas ocorridas durante o desenvolvimento das plantas, sobretudo no período de maturação de frutos. Stevens \& Rick (1986) também relatam que obter sucesso numa seleção de progênies em uma população segregante é difícil, por causa do impacto ambiental no conteúdo de sólidos, sendo que, muitas vezes a seleção em populações segregantes não é efetiva porque as variações na irrigação, textura do solo, resistência a doenças, podem ter grandes efeitos sobre o conteúdo de sólidos. Neste sentido, Prieto et al. (1999) obtiveram resultado que indica que o uso de estratégias de irrigação produzindo um moderado déficit hídrico, durante o período final de maturação dos frutos, melhorou o valor do brix, principalmente quando utilizou-se irrigação por gotejamento. Silva et al. (1999) obtiveram alta concentração de sólidos solúveis (de 4,97 a $5,71 \%$ ) testando 6 cultivares de tomate para processamento, em Brasília, sob irrigação por gotejamento subsuperficial, e creditaram esses valores ao correto ma- nejo da irrigação, principalmente durante a maturação dos frutos. Mas, no presente experimento, os genótipos com as melhores FC, apresentaram os menores ${ }^{\circ}$ Brix (Tabelas 2, 3 e 5). Neste sentido, Amaral Jr. et al. (1997) relatam que ganhos genéticos simultâneos poderiam ser alcançados de forma mais efetiva entre número total de frutos e teor de sólidos solúveis, já que essas duas características foram mais fortemente correlacionadas genotipicamente, com valor de 64,74\%. Entretanto, Cruz \& Regazzi (1997) alertam para o fato de que correlações genotípicas são próprias de cada caráter e população, não sendo recomendada a extrapolação, o que talvez explique o fato de, neste ensaio, ter-se obtido correlações fenotípica e genética negativas entre essas duas variáveis.

Para REND, as correlações fenotípica e genotípica foram positivas com PT, FC, ${ }^{\circ}$ Brix, COR, NFMP e pH e negativa somente com ACID. Desta forma, com base nos resultados desse ensaio, pode-se inferir que seria interessante selecionar as famílias baseando-se, principalmente, na variável rendimento industrial, pois não haveria prejuízo para as demais características desejadas, com exceção à acidez. Não seria interessante a utilização da variável FC por esta apresentar correlações fenotípica e genética negativas com o ${ }^{\circ}$ Brix.

Com base nos critérios acima descritos conclui-se que os genótipos que apresentaram melhor desempenho nas condições deste experimento foram UFU 06, Heinz 7155N2, Heinz 9553 e Hypeel 108.

\section{AGRADECIMENTOS}

Ao pesquisador Leonardo Fontes, Unilever Bestfoods, por fornecer a área experimental, materiais, equipamentos e a mão-de-obra necessários para a realização deste trabalho.

\section{LITERATURA CITADA}

AMARAL JÚNIOR, A.T.; CASALI, V.W.D.; CRUZ, C.D.; FINGER, F.L. Correlações simples e canônicas entre caracteres morfológicos, agronômicos e de qualidade em frutos de tomateiro. Horticultura Brasileira, Brasília, v. 15, n. 1, p. 49-52, 1997. 
CARVALHO, J.O.M. Seleção de famílias de tomateiro para processamento nos sistemas pivô central e gotejamento. 2002. 113 p. (Tese mestrado), ICIAG, UFU, Uberlândia.

CERNE, M.; RESNIK, M. Fruit quality of tomato cultivars. Acta-Horticulturae, n. 376, p. 313-318, 1994. CINTRA, A.A.D.; GRILLI, G.V.G.; BRAZ, L.T.; SANTOS, G.M.; BRAZ, B.A. Caracterização de frutos de cultivares de tomateiro para processamento. Horticultura Brasileira, Brasília, v. 18, Suplemento, p. 723-725, 2000.

CONCEIÇÃO, F.A.D. Características agronômicas e industriais de cultivares de tomateiro (Lycopersicon esculentum Mill.) de porte determinado, em duas épocas de cultivo. 1981. 142 p. (Tese), UNESP, Botucatu.

CRUZ, C.D. Programa GENES - Aplicativo computacional em genética e estatística. Viçosa: Editora UVF, 1997. 442 p.

CRUZ, C.D.; REGAZZI, A.J. Modelos Biométricos Aplicados ao Melhoramento Genético. Viçosa: Editora da UFV, 1997. 390 p.

FERREIRA, D.F.; MUNIZ, J.A.; AQUINO, L.H. Comparações múltiplas em experimentos com grande número de tratamentos - utilização do teste de Scott-Knot. Ciência Agrotécnica, v. 23, n. 3, p. 745-752, 1999.

FILGUEIRA, F.A.R. Novo Manual de Olericultura - Agrotecnologia moderna na produção e comercialização de hortaliças. Viçosa: Editora UFV, 2000.

FONSECA, H. Tomate - pré-processamento. In: Minami, K.; Fonseca, H. Tomate-produção, préprocessamento e transformação agroindustrial. Série Extensão Rural $n^{\circ}$ 8. Piracicaba: FEALQ, $1982.92 \mathrm{p}$.

GIORDANO, L.B.; SILVA, J.B.C; BARBOSA, V. Escolha de cultivares e plantio. In: Silva, J.B.C., Giordano, L.B. (Org.). Tomate PAra processamento industrial. Brasília: EMBRAPA Comunicação para Transferência de Tecnologia / EMBRAPA Hortaliças, 2000. 168 p.

GOMES, F.P. Curso de estatística experimental. $13^{\mathrm{a}}$ ed. Piracicaba: Nobel, 1990. 468 p.
JULIATTI, F.C.; DINIZ, F.C.V.; BARBIZAM, E.L.; CARDOSO, K.; LUZ, J.M.Q.; CARVALHO, J.O. M. Avaliação de famílias $\mathrm{F}_{5}$ de tomateiro grupo agroindustrial, plantio de inverno, Uberlândia - MG. Horticultura Brasileira, Brasília, v. 19, Suplemento 2, 2001. CD-ROM. Trabalho apresentado no $41^{\circ}$ Congresso Brasileiro de Olericultura, 2001.

MACUA, J.I.; GARNICA, J.; MERINO, J.; GUTIERREZ, M. Processing tomato variety choice in the Ebro Valley. Acta-Horticulturae, Pamplona, Spain, n. 487, p. 339-342, 1999.

MALUF, W.R. Melhoramento genético do tomate (Lycopersicon esculentum mill.). $199417 \mathrm{p}$ (Apostila). Depto. de Agricultura - UFLA, Lavras. MELO, P.C.T. A cadeia agro-industrial do tomate no brasil: retrospectiva da década de 90 e cenários para o futuro. Horticultura Brasileira, Brasília, v. 19, n. 2, julho 2001. Suplemento 2. CD-ROM. Palestra apresentada no $41^{\circ}$ Congresso Brasileiro de Olericultura, 2001.

NAKAMAE, I.J.; PASTRELlO, C.P. (Ed.) Agrianual 1999 - Anuário da Agricultura Brasileira. São PAulo: Editora Argos Comunicação. 1999. p. 489-497.

NAKAMAE, I.J.; PASTRELlO, C.P. (Ed.) Agrianual 2000 - Anuário da Agricultura Brasileira. São Paulo: Editora Argos Comunicação. 2000. p. 515-526.

PHENE, C.J. Efficient irrigation systems and irrigation scheduling for processing tomato: the challenge. Acta-Horticulturae, Pamplona, Spain, n. 487, p. 479-485, 1999.

PRIETO, M.H.; BALLESTEROS, R.; LÓPEZ, J. Influence of irrigation system and strategy on the agronomic and quality parameters of the processing tomato in extremadura. ActaHorticulturae, Pamplona, Spain, n. 487, p. $575-$ 579. 1999.

RAMALHO, M.A.P.; SANTOS, J.B.; ZIMMERMANN, M.J.O. Genética Quantitativa em Plantas Autógamas - Aplicações ao melhoramento do feijoeiro. Goiânia: Editora da UFG, 1993. $271 \mathrm{p}$.
RESENDE, G.M.; COSTA, N.D. Produtividade de tomate industrial no Vale do São Francisco. Horticultura Brasileira, Brasília, v. 18, n. 2, p. 126-129, 2000.

RICK, C.M. The tomato. Scientific American, v. 239, n. 2, p. 67-76, 1978.

SCOTT, A.J.; KNOTT, M. A cluster analysis method for grouping means in the analysis of variance. Biometrics, v. 3, n. 30, p. 507-512, 1974. SILVA, J.B.C.; GIORDANO, L.B.; BOITEX, L.S.; LOPES, C.A.; FRANÇA, F.H.; SANTOS, J.R.M.; FURUMOTO, O.; FONTES, R.R.; MAROUELLI, W.A.; NASCIMENTO, W.M.; SILVA, W.L.C.; PEDREIRA, W. Cultivo do tomate (Lycopersicon esculentum Mill.) para industrialização. Brasília: Embrapa Hortaliças, 1994. p. 6-7.

SILVA, W.L.C.; GIORDANO, L.B.; MAROUELLI, W.A.; FONTES, R.R.; GORNAT, B. Response of six processing tomato cultivars to subsurface drip fertigation. Acta-Horticulturae, Pamplona, Spain, n. 487, p. 569-573, 1999.

SILVA, W.L.C.; MAROUELLI, W.A. Exploratory studies on microirrigation for processing tomatoes in Central Brazil. In: INTERNATIONAL MICROIRRIGATION CONFERENCE, 5., 1995, Orlando. Proceedings. Orlando: ASAE, 1995, p. 904-908.

STEEL, R.G.D.; TORRIE, J.H. Principles and procedures of statistics: a biometrical approach. New York: McGraw - Hill Book Company, 1980. $633 \mathrm{p}$.

STEVENS, M.A.; RICK, C.M. Genetics and breeding. In: ATHERTON, J.G., RUDICH, J. (Ed.) The tomato crop - A scientific basis for improvement. London: Chapman and Hall, 1986. p. 35-109.

ZAHARA, M. Influence of plant density on yields of process tomatoes for mechanical harvest. Journal of American Society of Horticultural Science, n. 94, p. 510-512, 1970. 